\title{
Motivos da não realização do pré-natal por gestantes
}

\author{
Reasons for non-completion of prenatal care by pregnant women
}

Razones para no realizar atención prenatal a mujeres embarazadas

Fernanda Lopes de Souza ${ }^{1 *}$, Samantha de Mello Saccol'1, Taila Carvalho de Aguiar Rolim¹, Cleci Lourdes Schmidt Piovesan-Rosanelli ${ }^{1}$, Denise Miranda Conterato ${ }^{1}$, Elenir Terezinha Rizzetti Anversa'.

\section{RESUMO}

Objetivo: Conhecer quais os motivos que levaram gestantes a não realizarem nenhuma consulta de pré-natal em um município da Região Central do Rio Grande do Sul, no ano de 2017. Métodos: Estudo de campo de abordagem qualitativa de natureza descritivo-exploratória. Os endereços das mulheres foram acessados por meio de consulta ao Banco de Dados do Sistema de Informação de Nascidos Vivos. Na sequência, foi realizada a busca ativa no domicílio. Participaram oito mulheres, por meio de entrevista semiestruturada. Análise de conteúdo seguiu os passos preconizados por Minayo, pré-análise, exploração do material, tratamento e interpretação dos resultados. Resultados: Emergiu-se três categorias de análise: "Fragilidade da rede de apoio social a mulher gestante"; "Fragilidades no cuidado com o "eu", com olhar para o cuidado com o bebê" e "Fragilidade nos processos de trabalho das equipes de saúde no cuidado a gestantes". Conclusão: Evidencia-se vários motivos para a não realização do pré-natal por parte das gestantes, estes envolvem atitudes e transcendem os aspectos biológicos, sendo que este estudo poderá subsidiar a academia, gestores e profissionais da saúde com vistas a assegurar as políticas públicas preconizadas à saúde da mulher, em especial no processo da gestação.

Palavras-chave: Assistência pré-natal, Atenção primária à saúde, Gestação, Cuidado.

\section{ABSTRACT}

Objective: To know the reasons that led pregnant women to not attend any prenatal consultation in a city in the Central Region of Rio Grande do Sul, in 2017. Methods: field study of qualitative approach of descriptiveexploratory nature. Women's addresses were accessed by consulting the Live Births Information System Database. Next, the active search at home was performed. Eight women participated through a semistructured interview. Content analysis followed the steps recommended by Minayo, namely pre-analysis, material exploration, treatment, and interpretation of results. Results: three categories of analysis emerged: "Fragility of the social support network for pregnant women"; "Weaknesses in caring for the 'self', regarding the baby care" and "Weakness in the work processes of health teams in pregnant women care". Conclusion: There are several reasons for the non-completion of prenatal care by pregnant women. They involve attitudes and transcend biological aspects. This study may subsidize the academy, managers, and health professionals in order to ensure public policies recommended for women's health, especially in the process of pregnancy.

Keywords: Prenatal care, Primary health care, Pregnancy, Care.

\section{RESUMEN}

Objetivo: conocer las razones que llevaron a las mujeres embarazadas a no realizar ninguna consulta prenatal en una ciudad de la Región Central de Rio Grande do Sul, en el año 2017. Métodos: estudio de campo con un enfoque cualitativo de naturaleza descriptiva-exploratoria. Se accedió a las direcciones de las mujeres consultando la base de datos del sistema de información sobre nacimientos vivos. Luego, se realizó la búsqueda activa en el hogar. Ocho mujeres participaron, a través de entrevistas semiestructuradas. EI análisis de contenido siguió los pasos recomendados por Minayo, preanálisis, exploración de materiales, tratamiento e interpretación de resultados. Resultados: surgieron tres categorías de análisis: "Fragilidad de la red de apoyo social para mujeres embarazadas"; "Debilidades en el cuidado del" yo ", al observar el cuidado

${ }^{1}$ Faculdade Integrada de Santa Maria (FISMA), Santa Maria - RS. *E-mail: fernanda_I.souza@hotmail.com 
del bebé" y "Debilidad en los procesos de trabajo de los equipos de salud en el cuidado de las mujeres embarazadas". Conclusión: hay varias razones por las cuales las mujeres embarazadas no realizan atención prenatal, estas implican actitudes y trascienden los aspectos biológicos, y este estudio puede apoyar a la academia, gerentes y profesionales de la salud para garantizar políticas públicas recomendado para la salud de la mujer, especialmente en el proceso de embarazo.

Palabras clave: Cuidado prenatal, Atención primaria de salud, Gestación, Cuidado.

\section{INTRODUÇÃO}

A Política Nacional de Atenção Integral à Saúde da Mulher (PNAISM) considerando algumas lacunas, desde a luta pela implantação do Programa de Assistência Integral à Saúde da Mulher (PAISM), em 1983, amparado pelo movimento feminista e o modo de redemocratização do país executam um papel crucial para a sua concepção e desenvolvimento, até a contemporaneidade quando se amplia e consolida o Estado Democrático no Brasil, incluindo na agenda nacional a saúde como direito e cidadania (BRASIL, 2004).

O pré-natal (PN) deve ser estruturado para atender as reais necessidades das gestantes por meio da aplicação dos conhecimentos técnico-científicos e recursos adequados e disponíveis para cada caso. As ações de saúde precisam estar fortalecidas, estruturadas e direcionadas à cobertura de toda a população alvo da área de alcance das unidades de saúde, a fim de proporcionar o seguimento no cuidado, o acompanhamento e a avaliação dessas ações sobre a saúde materna-perinatal (BRASIL, 2011).

O Ministério da Saúde (2011), vem desenvolvendo programas e legislações legais para a normatização da assistência ao PN e nascimento como o Programa de Humanização no Pré-natal e Nascimento (PHPN) e a Rede Cegonha $(R C)$ que disponibiliza ações para garantir 0 atendimento de qualidade, seguro e humanizado para todas as mulheres. Com ênfase desde o planejamento reprodutivo, passando pelas fases da confirmação da gravidez, do pré-natal, pelo parto, puerpério e primeira infância.

A gestação é um momento de grande mudanças, descobertas e aprendizados, sendo uma oportunidade para os profissionais de saúde investirem em estratégias de educação e cuidado em saúde permanente, visando o bem-estar da mulher e da criança, assim como a inclusão do pai e/ou parceiro e família, desde que esse seja o desejo da mulher, a fim de proporcionar a integralidade do cuidado.

Abordando sobre a questão dos direitos sexuais, sociais, trabalhistas, desejo e planejamento da gestação acolhendo-a de forma singular e única, proporcionado o olhar para a detecção precoce de problemas (BRASIL, 2012; BRASIL, 2016).

Gonçalves CV, et al. (2009), descrevem que são inquestionáveis os benefícios propiciados pela atenção ao PN baseado em evidências cientificas para a redução da morbimortalidade de mulheres e seus filhos. Visto que, mesmo com todos os avanços tecnológicos e benefícios do PN, há uma parcela de gestantes que ainda não realizam nenhuma consulta.

Diante das assertivas o estudo teve como objetivo conhecer os motivos que levaram as gestantes á não realizarem nenhuma consulta de PN em um município da região central do estado do Rio Grande do Sul no ano de 2017.

\section{MÉTODOS}

Estudo de campo com abordagem qualitativa de natureza descritiva-exploratória (MINAYO MCS, 2013). Acessado o setor de Vigilância Epidemiológica (VE) de um município da região central do Estado do Rio Grande do Sul, no mês de agosto de 2018 para verificar o quantitativo de gestantes que não realizaram nenhuma consulta de PN no ano de 2017, buscando-se subsídios na Declaração de Nascidos Vivos (DNV).

A partir das informações da localização das gestantes, foi organizado e classificado os endereços por região administrativa a fim de facilitar a busca das informações inerentes ao objeto deste estudo. Com base da posse destas informações a pesquisadora realizou a busca ativa através do endereço da DNV das mulheres, em suas residências na área urbana, no período de setembro de 2018. 
Participaram do referido estudo oito mulheres. No entanto, destacam-se dificuldades na localização de endereços conforme a DNV, devido à mudança de endereço das mesmas, bem como a indisponibilidade de acesso nas primeiras tentativas de contatos e a uma parcela de mulheres, por motivos de internação para tratamentos decorrentes de uso de substâncias psicoativas.

Critérios de inclusão: mulheres que não realizaram nenhuma consulta de PN no ano de 2017, conforme as informações registradas na DNV, adstritas a área urbana do município, mulheres que apresentavam condições cognitivas para responder a entrevistas e que foram convidadas a participar da mesma e as que aceitaram e assinaram o Termo de Consentimento Livre e Esclarecido (TCLE). Os critérios de exclusão consistiram de mulheres que realizaram as consultas de PN e pessoas com dificuldades cognitivas de compreensão para responder a entrevista e residir na área rural do município.

Foi aplicada entrevista semiestruturada com questões relativas a aspectos sociodemográficose questões abertas norteadoras visando explorar os possíveis motivos que induziram as mesmas a não realização do PN. As entrevistas foram gravadas e transcritas na sua integralidade e elaborado um quadro a fim de organizar as respostas das voluntárias para facilitar a análise dos dados e posterior categorização.

As entrevistas foram coletadas por meio de um gravador digital, visando respeitar a fidedignidade das falas na análise dos principais assuntos que emergiram. Com vistas à validação das informações, ao final das entrevistas, as respondentes tiveram oportunidade de escutar seu depoimento e alterar ou confirmar algum aspecto que julgasse pertinente.

A análise dos dados foi realizada conforme pressupostos de Minayo MCS (2013). Após a leitura exaustiva, foram identificados os núcleos de sentido relativos a motivos que levaram as mulheres a não realizarem nenhuma consulta de PN. Estabeleceu-se três categorias: Fragilidade da rede de apoio social a mulher gestante - trata-se das dificuldades da rede de apoio social a mulher gestante relativa a fatores de âmbito familiar, individual e social.

Fragilidades no cuidado com o "eu", com olhar para o cuidado com o bebê - trata dos aspectos relativos à educação em saúde ao identificar o desconhecimento de gestantes sobre a gestação onde se visualiza a pouca importância do seu cuidado a gestante e transfere o cuidado a seu filho. Fragilidades nos processos de trabalho das equipes de saúde no cuidado a gestantes - discute como as mulheres que não realizam consulta significam o cuidado do PN.

Para manter o anonimato dos sujeitos da investigação foi utilizada codificação pela letra $M$ (Mulher) seguida de números ordinais em ordem crescente. O Projeto de pesquisa foi aprovado pelo Comitê de Ética da Universidade Federal de Santa Maria (UFSM) CAAE: 93974518.3.0000.5346, sob o Parecer Consubstanciado número: 2.821.268.

\section{RESULTADOS}

\section{Caracterização das mulheres que não realizaram nenhuma consulta de pré-natal}

Atendiam aos critérios de inclusão oito mulheres, com idade entre 16 a 45 anos, sendo uma adolescente, a maioria de cor branca, solteiras, com companheiros, quatro com ensino superior incompleto, uma com ensino superior completo, duas com ensino médio e uma com ensino fundamental. Tinham o cuidado do lar como principal ocupação. Quanto ao número de filhos anteriores constatou-se de um número de um a nove filhos, a maioria eram multíparas e apenas uma era primípara. Destas, seis realizaram o parto vaginal e duas o parto cesáreo. No momento das entrevistas, sete estavam amamentando, entretanto, nenhuma planejou a gestação. A maioria residia próxima a Unidade Básica de Saúde (UBS), e possuíam facilidade de chegar até o serviço de saúde.

\section{Categoria 1 - Fragilidade da rede de apoio social a mulher gestante}

Percebem-se na alocução das respondentes que os motivos da não realização do PN está associado à precariedade do suporte social prestado pela família, amigos ou vizinhos que visam contribuir para o cuidado das gestantes a aceitação da gestação e a violência sofrida. 
“... não realizei o $P N$, eu não tinha com quem deixar os meus dois filhos pequenos ... os dois bebês menores ..." M2

"Porque eu tinha dois bebês e não tinha com quem deixar, assim não tive como ir fazer as consultas de PN... As minhas irmãs trabalhavam ... Eu tive dificuldades de ir até o posto, por isso não fiz o PN. Não pela localização do posto, e sim de como me locomover com eles. Imagina ficar lá com dois bebês, mais a barriga, ficar aguardando isso é difícil". M3

“... aconteceram alguns problemas familiares, eu me mudei também, acabei indo para fora. Faleceu um parente, no caso, do meu ex-marido, pai do meu filho, foi meio conturbado. Deu tudo certo, como era meu segundo filho, já sabia mais ou menos como e o que era pré-natal, mas claro, os exames de praxe mesmo, que era para terem sido feitos, eu acabei não fazendo. Aconteceram alguns problemas familiares, a mudança de casa, eu não fiz o PN". M5

"Por que eu escondi a gravidez de minha família. Eu tinha medo dos meus pais não aceitarem a minha gravidez. Meu namorado, dizia para eu não contar, mas um dia a barriga ia aparecer..., mas, mesmo assim escondi, até quando pude. Eu usava roupas bem largas para meus pais não perceberem a barriga" M1

"Eu engravidei de um estupro, entendeu... Eu não quis isso, eu fui muito agredida, foi um assalto, eu não quis. O bebê foi para adoção, não está comigo, eu rejeitei aquela criança, eu não queria a gestação ... eu não havia falado que tinha sido um estupro..., eu fiquei muito doente, eu não queria essa gestação, eu não me alimentava. Tive depressão" M8

\section{Categoria 2 - Fragilidades no cuidado com o "eu", com olhar para o cuidado com o bebê}

Quando questionadas sobre o conhecimento da importância de realizar as consultas do PN, para uma parcela de mulheres, o desconhecimento de sinais e sintomas da gravidez, o cuidado transferido para a figura do filho que estar por vir, o não planejamento reprodutivo, bem como as contingências relativas ao trabalho e a falta de insight sobre o papel da mesma, na busca de garantia para a saúde do filho, são razões para não realização do PN.

"Considero muito importante o PN para fazer todos os exames e ultrassons, para ver se está tudo bem com a criança”. M1

“... Para saber se a criança está bem ou não”. M2.M4

“... É importante para saber a situação do bebê, para saber como ele está, mas eu não fiz". M5, $M 7$

“... Para saber se a mãe tem doença para não passar para o bebê”. M6

"... é que eu sou o homem da casa. Eu não tinha certeza que estava grávida, depois com o passar do tempo já estava perto de ganhar. Em seguida eu ganhei quando minha barriga apareceu, como eu não tinha bem certeza, porque barriga eu sempre tive, e não se mexia nem nada, eu fui empurrando ... empurrando ... quando apareceu a barriga eu já estava quase ganhando". M7

“... Sim. Porque eu fui diagnosticada, no caso eu estava com uma ..., me fugiu agora o nome agora, um corrimento. Eu tinha só o medicamento que o médico havia me passado, mas não resolveu o corrimento, aí fiz o exame, como demorou muito, eu não sabia que estava gravida, eu fui deixando. Achava que era do corrimento, pelos sintomas que sentia. Enjoo, ardência para fazer xixi, as vezes náuseas ...". M4

“... dela eu não fiz de preguiça mesmo, de preguiçosa. Não teve nenhum outro motivo, sou bem sincera, foi de preguiça mesmo ...". M7 
"Como eu vou dizer, quando eu tive meu gurizinho, eu não fiz o PN, porque eu trabalhava de cuidadora de idosos ..., eu não tinha tempo, nem para mim ...". M7

“... eu trabalhava direto, não tinha tempo de ir no posto, depois do nascimento que eu parei de trabalhar ...".M6

\section{Categoria 3 - Fragilidades no processo de trabalho das equipes de saúde}

As falas das participantes remetem as fragilidades dos dispositivos da Política Nacional de Humanização (PNH) e desvalorização de tecnologias leves pelas gestantes.

"Eu cheguei no postinho e só que na época não tinha ginecologista, não estavam agendando mais na época, eu até tenho plano privado, só que não tinha condições de pagar a consulta, estava apertada ...". M5

"Procurei sim, para descobrir e confirmar se estava gravida, fiz o teste de farmácia em casa e queria ter o teste de confirmação, fui fazer o exame de sangue e deu positivo, não voltei mais". M8

"... os médicos não fazem nada no PN, tão ali, só conversam contigo e te mandam embora. Eu nunca fiz um exame de toque ...". M3

"... fui no postinho para autorização do transvaginal. Demorou o resultado dos exames, daí fui deixando". M4

\section{DISCUSSÃO}

Rosa CQ, et al. (2014) e Rocha IMS, et al. (2017), identificaram associação a não realização de PN, mulheres com baixa escolaridade, principalmente para aquelas com menos de quatro anos de estudo, solteiras e multíparas, e adolescentes. O que contrapõem com os achados do presente estudo sendo que a não realização do PN esteve associada às mulheres com ensino superior incompleto, estes achados podem estar conexos ao ano de realização do estudo, uma vez que as mulheres estão buscando mais a formação profissional.

Pelas falas das mulheres, evidenciou-se dificuldades para realização do $\mathrm{PN}$, sendo necessário repensar a rede de apoio social. A Rede de Apoio Social pode-se incluir a família, os amigos, relações comunitárias, serviços de saúde. Até mesmo a relação ocasional tem-se mostrado necessárias em todas as fases da gestação, no puerpério e no retorno da mãe ao trabalho.

Oliveira MR e Dessen MA (2012), enfatizam a rede de apoio como estratégia positiva para desfechos favoráveis na maternidade durante a gestação e o nascimento dos filhos. É necessário (re)significar o apoio emocional e práticas junto aos pais e familiares e comunidade para que sejam fortalecidos durante o processo de gestação, o que possibilitará lidar melhor com as necessidades advindas das gestantes proporcionado 0 cuidado desta com o nascimento dos filhos.

Schwartz T, et al. (2011), apontam que a relação da rede de cuidados extrafamiliar, que abarca também os serviços de saúde, é deficitária e gera obstáculos psicossociais, inserindo a necessidade de maiores investimentos dos profissionais da $A B$ em grupos de cuidado social, que influenciam no desenvolvimento saudável do binômio mãe-bebê. Assim, para Rosa CQ, et al. (2014) é essencial que as gestantes possam contar com apoio de familiares, amigos, vizinhos para dedicar-se a gestação. No entanto, a pesquisa nos mostra por meio dos relatados das mulheres, que a não realização do PN está ligada a falta de apoio social.

Mulheres deixaram de realizar consultas por não ter como atribuir o cuidado dos filhos, mesmo que pontualmente, sendo necessária a discussão de uma rede de apoio materna fortalecida. As contingências relativas aos fatores laborais foi outro motivo identificado que implicou na não adesão do PN. Destaca-se que é assegurada a toda gestante empregada o direito à estabilidade no emprego, desde a confirmação da gravidez até cinco meses após do parto. Sendo que a confirmação da gravidez deve ser atestada por meio do exame laboratorial e médico (BRASIL, 2012). Estudos evidenciam que a atenção PN pautada na qualidade do cuidado é capaz de reduzir a morbimortalidade materno infantil (LANSKY S, et al., 2014). 
Outra questão destacada no presente estudo foi a gravidez não planejada, fato que corrobora com um estudo que evidenciou a elevada prevalência de gravidez não planejada no Brasil (VIELLAS EF, et al., 2014). O estudo continua destacando que a principal causa de realização do PN tardio foi justificada por problemas pessoais e de barreiras de acesso o que contrapõem a nossa investigação, uma vez que as entrevistas residiam próximas as UBS.

Problemas pessoais foram citados com maior constância por mulheres sem companheiro, que não queriam engravidar, insatisfeitas com a gravidez ou que tentaram interromper a gestação. As primigestas referiram à razão "não saber que estava grávida" por ter iniciado o PN tardiamente (VIELLAS EF, et al., 2014).

Apesar dos incentivos legais e benefícios do planejamento familiar alicerçado nos direitos sexuais e reprodutivos dos casais, ainda há uma demanda contraceptiva não atendida, sendo necessário uma melhor compreensão dos intricados motivos que levam na atualidade mulheres em fase produtiva a não usarem nenhum método contraceptivo (FERREIRA ALC e SOUZA AI, 2018).

Acrescido aos motivos já discutidos pode-se salientar que a concepção enquanto resultado da violência sexual, contribui como fator para a não realização do PN. A violência sexual sofrida pela mulher decorrente de estupro acarreta problemas de ordem física, social e psicológica que afeta sua vida e o desconhecimento da legislação que assegura 0 abortamento legal e 0 respeito sem julgamento relativo à sua decisão (SCHWARTZ T, 2011)., colaboram para levar a gestação a termo, no entanto não cuidam de si por não ter sido concebido por meio do seu desejo (RIBEIRO SVO, et al., 2019).

A vítima de estupro vivencia uma situação complicada que é a violência sexual, seria ainda mais crítico obrigar a vítima a gerar o fruto de um crime (ROMAGNOLI J, 2019). A violência física bem como o ato sexual forçado são as duas modalidades mais comum de violência, e pode ocorrer na infância, adolescência, na fase adulta. A violência sexual pode trazer reflexos de ordem psicológica, como o medo e a depressão. Descobrir estar grávida após um estupro é uma condição que agride ainda mais a mulher.

Estudo realizado constatou que os sintomas depressivos estavam mais presentes na gestação quando a gestante era vítima de violência, seja ela psicológica ou física durante a gestação, contribuindo para o aparecimento de sintomas de depressão pós-parto (RIBEIRO SVO, et al., 2019). Outro motivo que levou a não realização do PN foi a pouca ou não importância dada pela mulher como forma de autocuidado no período gestacional mesmo marcado por grandes mudanças na vida da mulher, sendo consideradas as consultas de PN para evitar doenças e agravos para o recém-nascido.

Estudo aponta que entre os fatores da não realização do PN está a escassa importância que a gestante tem para com seu cuidado nesta etapa de vida, muita pela escassez de informações educação em saúde, como ferramenta para o cuidado (ROSA CQ, et al., 2014). As gestantes procuram PN pela importância préconcebida e idealizada ao longo do tempo, bem como pela obrigatoriedade imposta por parentes ou amigos, e não por uma demanda de pertencimento e significado da gestante (MARTINS QPM, et al., 2015).

Foram perceptíveis fragilidades no processo de trabalho da equipe, uma vez que a gestante não consegue identificar suas necessidades frente ao processo de trabalho instituído pela equipe de saúde, uma vez que há necessidade de se repensar o cuidado da perspectiva da integralidade e equidade balizado pelos princípios da Política Nacional de Humanização e potencializando a singularidade e protagonismo da gestante, uma vez que o PN, no seu âmbito de assistência, é obrigatório para toda gestante desde a concepção, sendo atribuição de o município dispor de um sistema próprio, organizado, para a assistência PN, no parto, no puerpério e neonatal, bem como a captação precoce e o acompanhamento das gestantes (BRASIL, 2012; BRASIL, 2016).

Acolhimento é uma tecnologia de cuidado que permite o direito ao acesso, atenção em tempo oportuno. Para qualificar a assistência, promover e ampliar relações humanas de forma horizontal, transversalmente em todos os encontros entre profissionais e usuários de forma democrática e solidária, na qual a gestante é o sujeito ativo no processo de produção da saúde. O acolhimento à gestante e do acompanhante tem função primordial na construção de vínculo de confiança com os profissionais e serviços de saúde, favorecendo seu protagonismo (BRASIL, 2014).

REAS/EJCH | Vol.Sup.n.55 | e3878 | DOI: https://doi.org/10.25248/reas.e3878.2020 Página 6 de 8 
Segundo percepções das gestantes acerca do cuidado recebido durante o PN deve estar pautado na qualidade, no acolhimento, e não apenas no número de consultas de PN o que não assegura qualidade, bem como satisfação das gestantes. $O$ acolhimento é um fato real, o que possibilita ver as gestantes a partir das suas necessidades alavancando estratégias de cuidado na atenção ao $\mathrm{PN}$, bem como no processo de organização dos serviços de saúde, através de mudanças de valores no processo de perfilhar as mulheres gestantes não só no campo da razão, mas também no campo do sentir, no qual, no ser humano, não há dissociação de corpo-mente-espírito (SILVA LA, et al., 2018).

Evidenciou-se que o cuidado do PN está centrado no papel do médico. Segundo Protocolo do MS preconiza que o acompanhamento de PN de risco habitual seja realizado por médicos generalistas, bem como o enfermeiro pode acompanhar inteiramente o PN de baixo risco na rede básica de saúde, conforme garantido pela Lei do Exercício Profissional, regulamentada pelo Decreto no 94.406/87. Entretanto, orienta que a consulta de PN de risco habitual seja intercalada com o profissional médico (BRASIL, 2017).

Estudo que analisou a consulta de enfermagem no PN constatou que o acolhimento se faz presente nos encontros, como forma de potencializar o cuidado, proporcionar organização dos serviços. Garantindo acesso, resolutividade e vínculo das gestantes com os profissionais, facilidade de agendamento de consultas de enfermagem às gestantes, vislumbrando a necessidade de mudanças no modelo biomédico para um novo modelo assistencial, centrado na promoção da saúde, prevenção de agravos e satisfação das gestantes com a consulta de enfermagem (GOMES CBA, et al., 2019).

\section{CONSIDERAÇÕES FINAIS}

É necessário que ocorra um realinhamento do mundo do trabalho por parte dos profissionais ao entenderem as motivações que levam gestantes não realizarem $\mathrm{PN}$, que vai muito além do âmbito biológico, fazendo-se necessário uma rede de proteção social fortalecida e o entendimento do significado da gestação sem juízo de valor, e que sejam acolhidas nos serviços potencializando as dimensões sociais, culturais, econômicas e familiares. O vínculo com as mulheres em todos os ciclos de vida com os profissionais de saúde é uma potência no cuidado, para que possam ter liberdade de expressarem a aceitação ou não da gestação e serem entendidas como forma de melhoria do cuidado e adesão ao PN.

\section{REFERÊNCIAS}

1. BRASIL. Ministério da Saúde. Portaria no 2.436 de 21 de setembro de 2017.Brasília: Diário Oficial. República Federativa do Brasil, 2017.

2. BRASIL. Ministério da Saúde. Protocolos da Atenção Básica: Saúde das Mulheres / Ministério da Saúde, 1. Atenção Básica. 2. Protocolos. 3. Saúde da Mulher. I. Título. II. Instituto Sírio-Libanês de Ensino e Pesquisa. Instituto SírioLibanês de Ensino e Pesquisa - Brasília: Ministério da Saúde, 2016.

3. BRASIL. Ministério da Saúde. Secretaria de Atenção à Saúde. Atenção ao pré-natal de baixo risco. Cadernos de Atenção Básica, $n^{\circ}$ 32. Secretaria de Atenção à Saúde. Departamento de Atenção Básica. - Brasília: Editora do Ministério da Saúde, 2012.

4. BRASIL. Ministério da Saúde. Secretaria de Atenção à Saúde. Departamento de Ações Programáticas Estratégicas. Política Nacional de Atenção Integral à Saúde da Mulher:princípios e diretrizes / Ministério da Saúde, Secretaria de Atenção à Saúde, Departamento de Ações Programáticas Estratégicas. - Brasília: Ministério da Saúde, 2004.

5. BRASIL. Ministério da Saúde. Secretaria de Atenção à Saúde. Departamento de Ações Programáticas Estratégicas. Manual de acolhimento e classificação de risco em obstetrícia / Ministério da Saúde, Secretaria de Atenção à Saúde, Departamento de Ações Programáticas Estratégicas. - Brasília: Ministério da Saúde, 2014.

6. BRASIL. Ministério da Saúde. Secretaria de Políticas de Saúde. Manual Prático para Implementação da Rede Cegonha. Brasília, 2011.

7. BRASIL. Ministério da Saúde. Secretaria de Políticas de Saúde. Violência intrafamiliar: orientações para prática em serviço / Secretaria de Políticas de Saúde. - Brasília: Ministério da Saúde, 2001.

8. FERREIRA ALCG, SOUZA AI. Demanda contraceptiva não atendida. Revista Brasileira de Saúde Materno Infantil. Recife, 2018; 18(4): 693-694.

9. GOMES CBA, et.al. Consulta de enfermagem no prénatal: narrativas de gestantes e enfermeiras. Revista Texto \& Contexto Enfermagem, 2019; 28: e20170544.

10. GONÇALVES CV, et al. Qualidade e equidade na assistência à gestante: um estudo de base populacional no Sul do Brasil. Caderno de Saúde Pública, 2009.

11. LANSKY S, et al. Pesquisa Nascer no Brasil: perfil da mortalidade neonatal e avaliação da assistência à gestante e ao recém-nascido. Caderno de Saúde Pública. 2014; 30 Suppl 1:S192-207. 
12. MARTINS QPM, et al Conhecimentos de gestantes no pré-natal: evidências para o cuidado de enfermagem. S A N A R E, Sobral, 2015; 14(02): 65-71.

13. MINAYO MCS. Pesquisa social: teoria, método e criatividade. 29. ed. Petrópolis, RJ: Vozes, 2013.

14. OLIVEIRA MR, DESSEN MA. Alterações na rede social de apoio durante a gestação e o nascimento de filhos. Estudos de Psicologia. Campinas. 2012; 29(1) - 81-88.

15. RIBEIRO SVO, et al. Violência e sintomas de depressão na gestação e materna na coorte BRISA: uma abordagem com modelagem de equações estruturais. Rev. Bras. Saúde Mater. Infant., Recife, 2019.

16. ROCHA IMS, et.al. Fatores que influenciam a não adesão ao programa de pré-natal. São Paulo: Revista Recien. 2017; $7(21): 21-29$.

17. ROMAGNOLI J. Aborto no caso de gravidez resultante de estupro: o prazo de escolha para a vítima. Revista Científica Doctum: Direito. DOCTUM. Caratinga. 2019; 1(3).

18. ROSA CQ, et al. Fatores associados à não realização de pré-natal em município de grande porte. Rev Saúde Pública. 2014; 48(6): 977-984.

19. SCHWARTZ T. Apoio social a gestantes adolescentes: desvelando percepções. Ciência\& Saúde Coletiva, 2011; 16(5): 2575-2585.

20. SILVA LA, et al. A humanização do cuidado pré-natal na perspectiva valorativa das mulheres gestantes. Rev Fun Care Online. 2018; 10(4):1014-1019.

21. VIELLAS EF, et.al. Assistência pré-natal no Brasil. Caderno de Saúde Pública, Rio de Janeiro, 2014; 30, supl. 1: S85S100. 